\title{
Modified INOvent for delivery of inhaled nitric oxide during cardiac MRI ${ }^{\text {ts }}$
}

\author{
Ganesh P. Devendra ${ }^{\mathrm{a}, \mathrm{b}}$, Stephen A. Hart ${ }^{\mathrm{a}, \mathrm{b}}$, Yuli Y. Kim ${ }^{\mathrm{a}, \mathrm{c}}$, Randy M. Setser ${ }^{\mathrm{d}}$, \\ Scott D. Flamm ${ }^{\text {a,d }}$, Richard A. Krasuski ${ }^{a, *}$ \\ ${ }^{a}$ Department of Cardiovascular Medicine, Heart and Vascular Institute, Cleveland, OH 44195, USA \\ ${ }^{\mathrm{b}}$ Cleveland Clinic Lerner College of Medicine, Cleveland, OH 44195, USA \\ ${ }^{\mathrm{c} C h i l d r e n ' s ~ H o s p i t a l ~ B o s t o n, ~ B o s t o n, ~ M A ~ 02115, ~ U S A ~}$ \\ ${ }^{\mathrm{d}}$ Department of Radiology, Imaging Institute, Cleveland, OH 44195, USA \\ Received 7 April 2011; revised 5 May 2011; accepted 6 May 2011
}

\begin{abstract}
Background: The aim of this study was to assess the feasibility of delivering NO through a modified system to allow clearance of the magnetic field and thus compatibility with cardiac magnetic resonance (CMR). Nitric oxide (NO) is an inhalational, selective pulmonary vasodilator with a wide range of applications in a variety of disease states, including diseases that affect the right ventricle. Accurate assessment of dynamic changes in right ventricular function necessitates CMR; however, delivery of NO is only possible using equipment that is not magnetic resonance imaging (MRI) compatible (INOvent delivery system, Ohmeda, Inc., Madison, WI, USA).

Methods: The INOvent delivery system was modified by using $35 \mathrm{ft}$. of standard oxygen tubing to allow NO delivery through an electrical conduit and into the MRI suite. The concentrations of oxygen $\left(\mathrm{O}_{2}\right)$, nitrogen dioxide (a harmful byproduct, $\left.\mathrm{NO}_{2}\right)$ and $\mathrm{NO}$ were measured in triplicate using the built-in electrochemical analyzer on the INOvent. After confirmation of safety, the system was used to administer drug to a patient $\mathrm{x}$, and dynamic MRI measurements were performed.

Results: When the standard INOvent was set to administer 40 ppm of NO, the mean/standard deviation of gas delivered was as follows: NO: 42/0 ppm; $\mathrm{NO}_{2}:$ 0.3/0.1 ppm; and $\mathrm{O}_{2}: 93 / 0$ ppm. In comparison, the gas delivery of the modified INOvent was follows: $\mathrm{NO}: 41 / 0$ ppm; $\mathrm{NO}_{2}$ : 0.5/0 ppm; and $\mathrm{O}_{2}: 93.7 / 0.6 \mathrm{ppm}$. During administration to an index patient with severe pulmonic insufficiency (PI), a measurable reduction in PI was observed by CMR.

Conclusions: Nitric oxide can be administered through $35 \mathrm{ft}$. of standard oxygen tubing without significantly affecting dose delivery. This technique has potential application in patients with right-sided structural heart disease for determination of dynamic physiological changes. (C) 2011 Elsevier Inc. All rights reserved.
\end{abstract}

Keywords: Modified INOvent; Inhaled nitric oxide; Cardiac MRI

\section{Introduction}

Cardiac magnetic resonance imaging (CMR) has emerged in recent years as the gold standard for detailed evaluation of

\footnotetext{
is Author disclosures: Dr. Krasuski is on the speaker's bureau of Pfizer Pharmaceuticals, Roche Pharmaceuticals, AGA Medical and United Therapeutics, and is both a consultant for and on the speaker's bureau of Actelion Pharmaceuticals.

* Corresponding author. Tel.: +1 2164457430 .

E-mail addresses: devendg@ccf.org (G.P. Devendra), harts2@ccf.org (S.A. Hart), yuli.kim@cardio.chboston.org (Y.Y. Kim), setserr@ccf.org (R.M. Setser), flamms@ccf.org (S.D. Flamm), krasur@ccf.org, krasusr@ccf.org (R.A. Krasuski).
}

the right ventricle (RV) [1-5]. CMR can be used to assess both morphological and functional aspects of the RV and pulmonary vasculature [6], and as such has a wide range of applications in right heart disease. These include, but are not limited to, evaluation of pulmonary hypertension, RV systolic dysfunction, RV diastolic dysfunction and rightsided valvular disease [7-12].

Nitric oxide (NO) is an inhaled selective pulmonary vasodilator that decreases pulmonary vascular resistance and is commonly used for vasodilator challenges in patients with pulmonary hypertension [13-17]. Considering the anatomic specificity of this vasodilator and its ability to reduce RV afterload, acute challenges with NO 
could measurably modify disease states outside of pulmonary hypertension and assess the potential reversibility of pathologic sequelae. In fact, NO has been shown to measurably reduce RV diastolic dysfunction [18] and could theoretically have similar effects on right-sided valve disease. Regardless of the disease state that is being tested, the ideal modality for functional imaging of the right ventricle during such a vasodilator challenge would certainly be CMR. Unfortunately, NO is typically delivered through a specialized delivery system (INOvent delivery system, Ohmeda, Inc., Madison, WI, USA) that has ferromagnetic components and therefore is not compatible with magnetic resonance imaging (MRI). The aim of this study was to determine the feasibility of administering NO in the CMR environment through a modified delivery system.

\section{Materials and methods}

This study was performed with approval by the local institutional review board, and the patient gave individual, signed informed consent. The study and manuscript are also in compliance with the provisions of the Health Insurance Portability and Accountability Act (HIPAA) of 1996. All data were compiled and analyzed by using the JMP 8.0 software (SAS Institute, Inc., Cary, NC, USA). Means were compared using two-sided $t$ tests and a $P$ value $\leq .05$ was considered significant.

\subsection{Modified NO delivery}

The standard method for NO delivery has been previously described [17]. Medical-grade NO gas (INO Therapeutics, Madison, WI, USA) was delivered via the INOvent delivery system (Fig. 1A) from source tanks and flushed through to the patient using supplemental oxygen $\left(\mathrm{O}_{2}\right)$ flow at $5 \mathrm{~L} / \mathrm{min}$. The dose of $\mathrm{NO}(\mathrm{ppm})$ is controlled by the INOvent regulator and confirmed by the electrochemical analyzer incorporated into the INOvent system (Fig. 1C) by connection to the sample port (Fig. 1B). The INOvent's electrochemical analyzer has been previously used to determine drug concentrations in modified iNO delivery methods [19]. Concentrations of NO (ppm), nitrogen dioxide (a harmful byproduct, $\left.\mathrm{NO}_{2}, \mathrm{ppm}\right)$ and $\mathrm{O}_{2}(\%)$ are continuously reported to the INOvent display from the analyzer (Fig. 1A).

As the INOvent is incompatible with the magnetic field in the MRI suite, we chose to extend the delivery tubing leading from the INOvent to the patient using five pieces of standard 7-ft. AirLife $\mathrm{O}_{2}$ tubing connected by AirLife $\mathrm{O}_{2}$ tubing connectors (Cardinal Health, Dublin, OH, USA) (Fig. 1D).
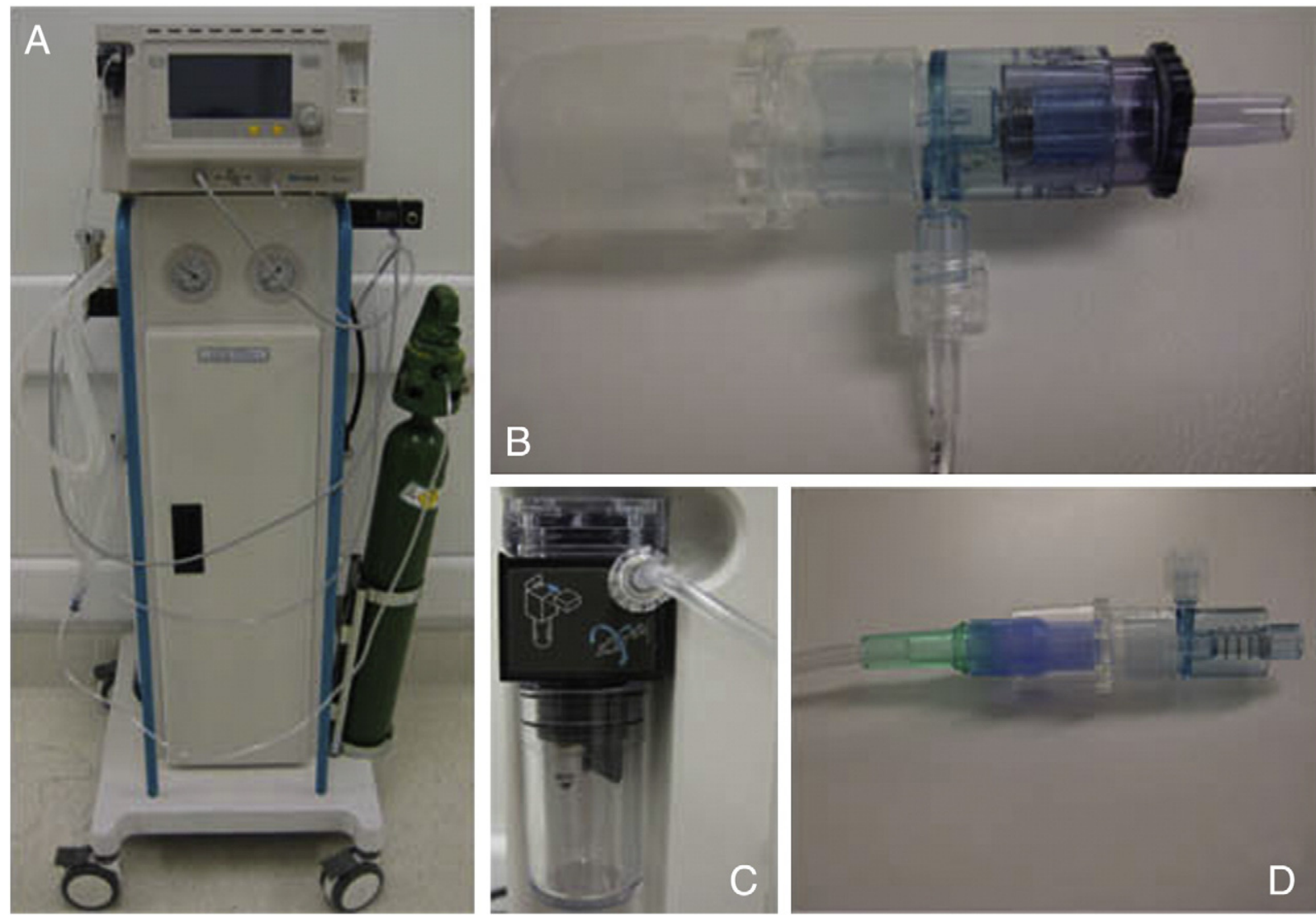

Fig. 1. INOvent delivery system (A), sample port (B), electrochemical analyzer (C) and modified sample port with $\mathrm{O}_{2}$ tubing (D). 
For the purpose of calibration, the tubing was looped back to INOvent and drug concentration was tested using the onboard electrochemical analyzer via factory-issued sampling line. For the purpose of drug administration to a patient, an extended delivery tube was routed through a shielded electrical conduit allowing the INOvent to remain safely outside the MRI suite.

To verify that the doses of delivered gases with the extended delivery tube were similar to the standard INOvent setup, we connected the electrochemical analyzer to the sample port at the end of the extended delivery tube (Fig. 1D). The INOvent regulator was set at standard doses of 10 , 20, 40 and $80 \mathrm{ppm}$ with an accompanying $\mathrm{O}_{2}$ flow of $15 \mathrm{~L} / \mathrm{min}$. The higher $\mathrm{O}_{2}$ flow rate was chosen in order to achieve adequate delivery through the extended tubing. Concentrations of delivered gas at each setting were measured in triplicate with both the standard and modified setups. Comparisons of $\mathrm{NO}, \mathrm{NO}_{2}$ and $\mathrm{O}_{2}$ at the various dosage settings between the standard and modified INOvent settings were analyzed using two-tailed $t$ tests of the means with a $P<.05$ assumed to be significant. For some measurements the range was too small to measure a standard deviation. As such, a $P$ value was not reported for all comparisons.

\subsection{Nitric oxide administration during CMR}

CMR imaging was performed using a 1.5-T unit (Magnetom Avanto, Siemens Medical Systems, Erlangen, Germany) with a multi-element phased-array surface coil. The study was performed with the patient in the supine position using a phased-array surface coil as a receiver and electrocardiographic gating. Images were obtained during end-expiratory breath-holds.

Initially, multiplanar scout images were obtained using a steady-state free-precession (SSFP) technique, followed by axial SSFP images encompassing the heart and great vessels. Cine SSFP images were then acquired in two long-axis orientations (vertical long-axis and four-chamber) and subsequently in short-axis orientation encompassing both ventricles. Typically, 12 to 16 contiguous short-axis cine SSFP images were acquired from the atrioventricular valve plane through the cardiac apex to measure ventricular volumes.

The SSFP sequence used for cardiac gated cine imaging had the following acquisition parameters: TR/TE/flip, 2.8 $\mathrm{ms} / 1.4 \mathrm{~ms} / 65^{\circ}$; field of view (FOV), $320 \mathrm{~mm}$; matrix, $160 \times 160$; slice thickness, $8 \mathrm{~mm}$; slice gap, $0 \mathrm{~mm}$; acquired voxel size (dimensions along the frequency, phase and slice encoding directions, respectively), $2.0 \times 2.0 \times 8.0 \mathrm{~mm}$, and the reconstructed voxel size in the corresponding directions, $1.3 \times 1.3 \times 8.0 \mathrm{~mm}$; and temporal resolution, $25-40 \mathrm{~ms}$, depending on heart rate.

Following cine imaging phase-contrast, a retrospectively gated, velocity-encoded phase-contrast sequence was performed perpendicular to the long axis of the main pulmonary artery just proximal to the pulmonary artery bifurcation with velocity-encoding initially set at $100 \mathrm{~cm} / \mathrm{s}$. If aliasing was noted, the velocity was progressively raised in $50 \mathrm{~cm} / \mathrm{s}$ steps until aliasing disappeared.

The sequence had the following acquisition parameters: TR/TE/flip $5.0 \mathrm{~ms} / 3.1 \mathrm{~ms} / 30^{\circ}$; FOV, 300-380 $\times 240-360$ $\mathrm{mm}$; matrix, 256×128 (typical in-plane resolution, $2.3 \times 1.3$ $\mathrm{mm}$ ); slice thickness, $6 \mathrm{~mm}$; number of signal averages, 1 ; and temporal resolution, $25-35 \mathrm{~ms}$, depending on heart rate. The typical breath-hold time ranged from 15 to $25 \mathrm{~s}$. Patients were encouraged to hold their breath during the whole acquisition. If obvious breathing artifacts were noted, the acquisition was repeated until artifacts were eliminated.

After completing the initial baseline CMR, the participant received $\mathrm{NO}$ at $40 \mathrm{ppm}$ through a non-rebreather mask connected to the modified INOvent with extended delivery tubing. This method of iNO delivery has an established safety record and is commonly used in acute testing with iNO $[16,20]$. After 4 min of $\mathrm{NO}$ administration (to achieve steady-state conditions), repeat cine imaging for ventricular volumes and function, and flow velocity mapping of the pulmonary valve were obtained identical to the baseline imaging.

Postprocessing and data measurements were completed off-line after transferring images to a commercially available workstation (Leonardo, Siemens Medical Solutions). There, $\mathrm{RV}$ volumes and function and pulmonary valve insufficiency were determined. Two independent readers made measurements with the average values between readers used.

From the stack of ventricular short-axis SSFP cine images, contours were drawn manually to delineate RV endo- and epicardial boundaries on the end-diastolic and end-systolic phases. From these, the end-diastolic (EDV) and end-systolic volumes (ESV) were computed and the following quantitative metrics derived: Stroke Volume $(\mathrm{SV})=\mathrm{EDV}-\mathrm{ESV}$; Ejection Fraction $(\mathrm{EF})=\mathrm{SV} / \mathrm{EDV}$; and Cardiac Output $(\mathrm{CO})=\mathrm{SV} * \mathrm{HR}$. For flow quantification data, the contours of the main PA were automatically traced on magnitude- and velocity-encoded images simultaneously with manual correction as needed. The following metrics were determined: forward flow volume, reverse (regurgitant) flow volume and regurgitant fraction calculated as [reverse flow volume/forward flow volume].

\section{Results}

\subsection{Nitric oxide delivery with modified INOvent setup}

Delivery of $\mathrm{O}_{2}$ was similar between the standard and modified INOvent setups at each setting (Table 1). At a

Table 1

Mean $\mathrm{O}_{2}$ delivery (\%) for the standard and modified INOvent

\begin{tabular}{lllll}
\hline & $10 \mathrm{ppm}$ & $20 \mathrm{ppm}$ & $40 \mathrm{ppm}$ & $80 \mathrm{ppm}$ \\
\hline Standard & $97 \pm 0$ & $96 \pm 0$ & $93 \pm 0$ & $88.7 \pm 0.6$ \\
Modified & $97 \pm 0$ & $96 \pm 0$ & $93.7 \pm 0.6$ & $89 \pm 0$ \\
$P$ value & - & - & .18 & .42 \\
\hline
\end{tabular}


Table 2

Mean $\mathrm{NO}_{2}$ delivery (ppm) for the standard and modified INOvent

\begin{tabular}{lllll}
\hline & $10 \mathrm{ppm}$ & $20 \mathrm{ppm}$ & $40 \mathrm{ppm}$ & $80 \mathrm{ppm}$ \\
\hline Standard & $0.2 \pm 0.2$ & $0.23 \pm 0.1$ & $0.33 \pm 0.1$ & $0.73 \pm 0.2$ \\
Modified & $0.27 \pm 0.2$ & $0.33 \pm 0.1$ & $0.5 \pm 0$ & $1.5 \pm 0$ \\
$P$ value & .69 & .1 & .04 & .02 \\
\hline
\end{tabular}

setting of $10 \mathrm{ppm} \mathrm{NO}$, mean delivered $\mathrm{O}_{2}$ was $97 \pm 0 \%$ in both the standard and modified INOvent. Similarly at the 20 -ppm setting, mean $\mathrm{O}_{2}$ was $96 \pm 0 \%$ with both the standard and modified INOvent setups. At a setting of $40 \mathrm{ppm}$ NO, delivered $\mathrm{O}_{2}$ in the standard setup yielded $93 \pm 0 \% \mathrm{O}_{2}$ and the modified INOvent delivered $93.7 \pm 0.6 \%(P=.18)$. At a setting of $80 \mathrm{ppm} \mathrm{NO}$, the standard INOvent delivered $88.7 \pm 0.6 \%$ $\mathrm{O}_{2}$ and the modified INOvent delivered $89 \pm 0 \%(P=.42)$.

$\mathrm{NO}_{2}$ levels were similar between the standard and modified INOvent setups (Table 2). At a setting of $10 \mathrm{ppm}$ $\mathrm{NO}$, the standard INOvent delivered $0.2 \pm 0.2 \mathrm{ppm} \mathrm{NO}_{2}$, while the modified setup delivered $0.27 \pm 0.2 \mathrm{ppm} \mathrm{NO}_{2}$ $(P=.69)$. At the 20-ppm NO setting, the standard INOvent delivered $0.23 \pm 0.1 \mathrm{ppm} \mathrm{NO}_{2}$, while the modified INOvent delivered $0.33 \pm 0.1 \mathrm{ppm} \mathrm{NO} \mathrm{NO}_{2}(P=.1)$. At the 40 -ppm setting, the standard INOvent delivered $0.33 \pm 0.1 \mathrm{ppm} \mathrm{NO}_{2}$ and the modified INOvent delivered $0.5 \pm 0 \mathrm{ppm} \mathrm{NO}_{2}(P=.04)$. At the 80 -ppm NO setting, the standard INOvent delivered $0.73 \pm$ $0.2 \mathrm{ppm}$ of $\mathrm{NO}_{2}$, whereas the modified INOvent delivered $1.5 \pm 0$ ppm NO $\mathrm{NO}_{2}(P=.02)$.

Finally, the mean delivery of NO was also similar between the standard and modified setups (Table 3). At the 10-ppm NO setting, the standard INOvent delivered $10.5 \pm 0.9 \mathrm{ppm}$ of $\mathrm{NO}$ and the modified INOvent delivered $10.1 \pm 0.9 \mathrm{ppm}(P=.62)$. At the 20 -ppm NO setting, the standard INOvent delivered $20.3 \pm 0.6 \mathrm{ppm}$ of $\mathrm{NO}$ and the modified INOvent delivered $20 \pm 0 \mathrm{ppm}$ NO $(P=.42)$. At the 40-ppm NO setting, the standard INOvent delivered $42 \pm 0 \mathrm{ppm} N O$ and the modified INOvent delivered $41 \pm 0$ ppm NO. At the 80-ppm NO setting, the unmodified INOvent delivered $86 \pm 0 \mathrm{ppm}$ of $\mathrm{NO}$, while the modified INOvent delivered $85 \pm 0 \mathrm{ppm}$.

\subsection{Evaluation of pulmonary valve insufficiency}

The patient undergoing evaluation was a 21-year-old male with a history of complete tetralogy of Fallot repair at age 5 with resultant severe pulmonary insufficiency (PI), undergoing preoperative evaluation of his right heart. His BMI was 23.6 and baseline blood pressure was 146/71, pulse

Table 3

Mean NO delivery (ppm) for the standard and modified INOvent

\begin{tabular}{lllll}
\hline & $10 \mathrm{ppm}$ & $20 \mathrm{ppm}$ & $40 \mathrm{ppm}$ & $80 \mathrm{ppm}$ \\
\hline Standard & $10.5 \pm 0.9$ & $20.3 \pm 0.6$ & $42 \pm 0$ & $86 \pm 0$ \\
Modified & $10.1 \pm 0.9$ & $20 \pm 0$ & $41 \pm 0$ & $85 \pm 0$ \\
$P$ value & .62 & .42 & - & - \\
\hline
\end{tabular}

Table 4

Cardiac magnetic resonance measurements

\begin{tabular}{lclc}
\hline & $\begin{array}{l}\text { Baseline } \\
\text { CMR }\end{array}$ & $\begin{array}{l}\text { CMR } \\
\text { with NO }\end{array}$ & $\begin{array}{l}\text { Difference } \\
(\%)\end{array}$ \\
\hline End systolic volume (ml) & 192 & 231 & 20 \\
End diastolic volume (ml) & 305 & 343 & 13 \\
Stroke volume (ml) & 113 & 112 & 1 \\
Heart rate (bpm) & 61 & 75 & 23 \\
Cardiac Output $(\mathrm{L} / \mathrm{min})$ & 6.9 & 8.4 & 22 \\
Regurgitant volume (ml) & 70 & 62 & -10 \\
Regurgitant fraction $(\%)$ & 47.7 & 42.0 & -12 \\
\hline
\end{tabular}

was 71 and oxygen saturation was $99 \%$ on room air. The patient's NYHA function class was II.

The baseline scan was performed without complications. Right ventricular analysis revealed an end diastolic volume of $305 \mathrm{ml}$ and an end systolic volume of $192 \mathrm{ml}$ for a stroke volume of $113 \mathrm{ml}$. The patient's heart rate was $61 \mathrm{bpm}$, yielding a cardiac output of $6.9 \mathrm{~L} / \mathrm{min}$. The pulmonary regurgitant volume was $70 \mathrm{ml}$ and the regurgitant fraction was 0.48 .

With $\mathrm{NO}$ administration, the right ventricular end diastolic volume increased to $343 \mathrm{ml}$ and the end systolic volume increased to $231 \mathrm{ml}$, giving an almost identical stroke volume of $112 \mathrm{ml}$. The heart rate modestly increased to 75 , yielding a cardiac output of $8.4 \mathrm{~L} / \mathrm{min}$. The regurgitant volume decreased to $62 \mathrm{ml}$ and the regurgitant fraction was decreased to 0.42 , representing a $12 \%$ reduction in PI (Table 4 ).

There were no complications associated with NO administration or performance of the additional images. The subject also reported no adverse effects when contacted on the day after the procedure.

\section{Discussion}

We have demonstrated that NO can be safely and efficaciously administered during CMR. We have also shown that selective pulmonary vasodilation can reduce afterload mismatch across a compromised pulmonary valve and in turn reduce the degree of pulmonary valve insufficiency. The importance of our findings is twofold: First, by demonstrating that NO may be delivered in an MRI suite, we have opened the door to dynamic functional assessment of the RV. This technique may also prove useful in other structural and congenital heart lesions, as well as in pulmonary arterial hypertension. Second, this study suggests that selective pulmonary vasodilation may prove to be a novel therapeutic target in pulmonary valve disease. In order to assess the latter we are currently enrolling patients into a larger study designed to more definitively evaluate the effects of NO inhalation on PI after tetralogy of Fallot repair.

Our measurements indicate that the delivery of NO through a standard INOvent and our modified INOvent are virtually identical across a wide range of doses. $\mathrm{O}_{2}$ delivery was similarly unchanged between the two delivery methods, 
although the $\mathrm{NO}_{2}$ level (a compound with potential pulmonary toxicity) was statistically increased with the modified INOvent setup at NO doses of 40 and 80 ppm. We hypothesize that the extended tubing increases the exposure time of $\mathrm{NO}$ (a highly reactive molecule) to $\mathrm{O}_{2}$ and would thereby facilitate oxidation of $\mathrm{NO}$ to $\mathrm{NO}_{2}$. The increased level of $\mathrm{NO}_{2}$ exposure during the CMR is well below the occupational exposure limit of 5 ppm averaged over an 8-h period [21] or the 3-ppm threshold suggested by INO Therapeutics and may pose no significant risk to patients. However, the accuracy of the $\mathrm{NO}_{2}$ measurement at $80 \mathrm{ppm}$ is not certain as levels may be too high to meet the criteria in the FDA guidance document [22]. Due to this issue, potential toxicity and the fact that the sampling port is not proximal to the subject mask in the modified, we would recommend against administering iNO through this method at the 80 -ppm setting as the $\mathrm{NO}_{2}$ increment was substantial. Using a lower dose should not influence the efficacy of this assay as doses as low as 20 or 40 ppm are standard protocol in acute vasodilator assessment [23].

We were able to demonstrate a $12 \%$ drop in pulmonary regurgitation with the administration of NO in our index patient. Although this reduction appears modest, the half-life of NO has been estimated between 2 and 6 s [24], while the breath-hold sequence necessary to complete this study was approximately $25 \mathrm{~s}$. The measured reduction in PI may therefore be much greater if continuous pulmonary vasodilation were achieved. These potential shortcomings should be evaluated in the future with a larger study cohort and longer-acting pulmonary vasodilators.

\section{Acknowledgments}

Joan Weaver, RT, and Angel Lawrence, RT, for expert technical help in performing the CMR examinations.

\section{References}

[1] Rathi VK, Biederman RW. Imaging of ventricular function by cardiovascular magnetic resonance. Curr Cardiol Rep 2004;6:55-61.

[2] Khaled A, Sven P, Holger T, Tim J, John PR, Mohan US. Normal human left and right ventricular dimensions for MRI as assessed by turbo gradient echo and steady-state free precession imaging sequences. J Magn Reson Imaging 2003;17:323-9.

[3] Beygui F, Furber A, Delepine S, Helft G, Metzger JP, Geslin P, et al. Routine breath-hold gradient echo mri-derived right ventricular mass, volumes and function: accuracy, reproducibility and coherence study. Int J Cardiovasc Imaging 2004;20:509-16.

[4] Maceira AM, Prasad SK, Khan M, Pennell DJ. Reference right ventricular systolic and diastolic function normalized to age, gender and body surface area from steady-state free precession cardiovascular magnetic resonance. Eur Heart J 2006;27:2879-88.

[5] Pennell DJ, Sechtem UP, Higgins CB, Manning WJ, Pohost GM, Rademakers FE, et al. Clinical indications for cardiovascular magnetic resonance (CMR): consensus panel report. J Cardiovasc Magn Reson 2004;6:727-65.

[6] McLure LER, Peacock AJ. Cardiac magnetic resonance imaging for the assessment of the heart and pulmonary circulation in pulmonary hypertension. Eur Respir J 2009;33:1454-66.
[7] Grapsa J, O’Regan DP, Pavlopoulos H, Durighel G, Dawson D, Nihoyannopoulos $\mathrm{P}$. Right ventricular remodelling in pulmonary arterial hypertension with three-dimensional echocardiography: comparison with cardiac magnetic resonance imaging. Eur J Echocardiogr 2009.

[8] Youssef A, Ibrahim el SH, Korosoglou G, Abraham MR, Weiss RG, Osman NF. Strain-encoding cardiovascular magnetic resonance for assessment of right-ventricular regional function. J Cardiovasc Magn Reson 2008;10:33.

[9] Papavassiliu T, Germans T, Fluchter S, Doesch C, Suriyakamar A, Haghi D, et al. CMR findings in patients with hypertrophic cardiomyopathy and atrial fibrillation. J Cardiovasc Magn Reson 2009;11:34.

[10] Myerson SG. Valvular and hemodynamic assessment with cmr. Heart Fail Clin 2009;5:389-400, vi-vii.

[11] Kuehne T, Yilmaz S, Steendijk P, Moore P, Groenink M, Saaed M, et al. Magnetic resonance imaging analysis of right ventricular pressure-volume loops: in vivo validation and clinical application in patients with pulmonary hypertension. Circulation 2004;110:2010-6.

[12] Menteer J, Weinberg PM, Fogel MA. Quantifying regional right ventricular function in tetralogy of Fallot. J Cardiovasc Magn Reson 2005;7:753-61.

[13] Griffiths MJD, Evans TW. Inhaled nitric oxide therapy in adults. N Engl J Med 2005;353:2683-95.

[14] Pepke-Zaba J, Higenbottam TW, Dinh-Xuan AT, Stone D, Wallwork J. Inhaled nitric oxide as a cause of selective pulmonary vasodilatation in pulmonary hypertension. Lancet 1991;338:1173-4.

[15] Frostell C, Fratacci MD, Wain JC, Jones R, Zapol WM. Inhaled nitric oxide. A selective pulmonary vasodilator reversing hypoxic pulmonary vasoconstriction. Circulation 1991;83:2038-47.

[16] Ichinose F, Roberts Jr JD, Zapol WM. Inhaled nitric oxide: a selective pulmonary vasodilator: current uses and therapeutic potential. Circulation 2004;109:3106-11.

[17] Wessel DL, Adatia I, Thompson JE, Hickey PR. Delivery and monitoring of inhaled nitric oxide in patients with pulmonary hypertension. Crit Care Med 1994;22:930-8.

[18] Gan CT-J, Holverda S, Marcus JT, Paulus WJ, Marques KM, Bronzwaer JGF, et al. Right ventricular diastolic dysfunction and the acute effects of sildenafil in pulmonary hypertension patients. Chest 2007;132:11-7.

[19] Platt DR, Swanton D, Blackney D. Inhaled nitric oxide (INO) delivery with high-frequency jet ventilation (HFJV). J Perinatol 2003;23: 387-91.

[20] Krasuski RA, Wang A, Harrison JK, Tapson VF, Bashore TM. The response to inhaled nitric oxide in patients with pulmonary artery hypertension is not masked by baseline vasodilator use. Am Heart $\mathrm{J}$ 2005;150:725-8.

[21] Markhorst DG, Leenhoven T, Uiterwijk JW, Meulenbelt J, van Vught AJ. Occupational exposure during nitric oxide inhalational therapy in a pediatric intensive care setting. Intensive Care Med 1996;22:954-8.

[22] Guidance for industry and for FDA reviewers. Guidance document for premarket notification submissions for nitric oxide delivery apparatus, nitric oxide analyzer and nitrogen dioxide analyzer. U.S. Department of Health and Human Services, Food and Drug Administration. Center for Devices and Radiological Health, Documents Issued on January 24; 2000.

[23] McLaughlin VV, Archer SL, Badesch DB, Barst RJ, Farber HW, Lindner JR, et al. ACCF/AHA 2009 expert consensus document on pulmonary hypertension. A report of the American College of Cardiology Foundation Task Force on expert consensus documents and the American Heart Association developed in collaboration with the American College of Chest Physicians; American Thoracic Society, Inc.; and the Pulmonary Hypertension Association. J Am Coll Cardiol 2009;53:1573-619.

[24] Moncada S. The 1991 Ulf von Euler lecture. The L-arginine: nitric oxide pathway. Acta Physiol Scand 1992;145:201-27. 